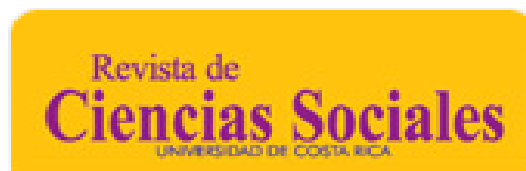

Revista de Ciencias Sociales (Cr)

ISSN: 0482-5276

revista.cs@ucr.ac.cr

Universidad de Costa Rica

Costa Rica

Méndez Bordón, Aliek; Rivas Diéguez, Aramís; Ramírez Pérez, Alexander ACCIONES PARA DISMINUIR LA FLUCTUACIÓN LABORAL Revista de Ciencias Sociales (Cr), vol. IV, núm. 146, 2014, pp. 27-33

Universidad de Costa Rica San José, Costa Rica

Disponible en: http://www.redalyc.org/articulo.oa?id=15340989003

- Cómo citar el artículo

- Número completo

- Más información del artículo

- Página de la revista en redalyc.org

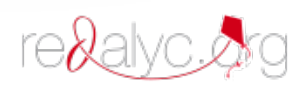

Sistema de Información Científica Red de Revistas Científicas de América Latina, el Caribe, España y Portugal Proyecto académico sin fines de lucro, desarrollado bajo la iniciativa de acceso abierto 


\title{
ACCIONES PARA DISMINUIR LA FLUCTUACIÓN LABORAL
}

\section{ACTIONS TO REDUCE LABOUR FLUCTUATIONS}

\author{
Aliek Méndez Bordón* \\ Aramís Rivas Diéguez ${ }^{* *}$ \\ Alexander Ramírez Pérez ${ }^{* * *}$
}

RESUMEN

\begin{abstract}
La inestabilidad laboral constituye una problemática que afecta a las organizaciones laborales. En este artículo se busca evaluar las causas de la fluctuación laboral en la Unidad Básica de Producción Cooperativa Luis Peña Martínez a través de métodos científicos y técnicas. Se identificó que los inadecuados métodos y estilos de dirección, así como, el bajo reconocimiento, constituyeron las problemáticas de mayor incidencia. Asimismo, el diseño de acciones mejoró el clima organizacional y los índices de fluctuación laboral en la entidad.
\end{abstract}

PALABRAS CLAVE: CUBA * TRABAJO * FLUCTUACIÓN LABORAL * MOTIVACIÓN

\section{ABSTRACT}

The labour instability is a problem that affects the labour organizations. This article seeks to evaluate the causes of labour fluctuation in the Basic Unit of Cooperative Production Luis Peña Martínez, through scientific methods and techniques. It was identified that inadequate methods and management styles and low recognition, were the problematics of higher incidence. Also the design of actions improved organizational climate and labor fluctuation rates in the institution.

KEYWORDS: CUBA * LABOUR * LABOUR FLUCTUATION * MOTIVATION

Centro Universitario Municipal Jesús Menéndez de la Universidad de Las Tunas "Vladimir Ilich Lenin” (ULT), Cuba. aliekmb@ult.edu.cu

** Centro Universitario Municipal Jesús Menéndez de la Universidad de Las Tunas "Vladimir Ilich Lenin" (ULT), Cuba. aramisrd@ult.edu.cu

*** Empresa Agropecuaria del Municipio Jesús Menéndez, Cuba. neomendez2014@gmail.com 


\section{INTRODUCCIÓN}

Las organizaciones laborales son agrupamientos humanos que se integran para cumplir actividades sistematizadas y reiteradas en torno a un sistema de objetivos establecidos en un espacio físico y con un carácter temporal perdurable (Moros y Díaz, 2005).

La actividad laboral es realizada por sujetos de los que depende el alcance de los objetivos de la organización, de la cual esperan bienestar y espacios de realización profesional. Este subsistema vincula al personal a su cometido funcional, a fin de mantener el tejido de comportamientos interrelacionados necesarios para el cumplimiento de los objetivos de la organización como tarea interdependiente (Rodríguez, 2012).

La conservación del personal en la organización es un proceso complejo, que implica un contrato entre el trabajador y la organización. La retención de los recursos humanos se refiere a la condición que hace a los individuos, que una vez reclutados, seleccionados, socializados y que han comenzado a trabajar en una organización, se mantengan dentro de ella. La preservación de los recursos humanos debe constituir un objetivo estratégico de la organización laboral, al permitir aprovechar las capacidades, destrezas y experiencias de los trabajadores en su propio desarrollo $y$ fomentar el sentimiento de pertenencia a ésta (Espinosa, Yanes, Espino, Abramo y Yáñez, 1999: s.d.).

El desarrollo y mejora de una organización a través de la planificación de las personas y la optimización de su aprovechamiento, es uno de los conceptos manejados en la actualidad y que produce mejores resultados, tanto en la eficiencia y calidad de los procesos y servicios desarrollados, como en lo relacionado con el perfeccionamiento intelectual de las personas y el aumento de su motivación y satisfacción en el trabajo.

Los retos que enfrenta la organización exigen que sus recursos humanos sean flexibles y creativos; por lo que debe realizarse una estrategia, convirtiéndose en un recurso estratégico para lograr una ventaja competitiva básica y real, más allá de los recursos materiales con los que se cuenta (Rodríguez, 2012).

En tal sentido, actualmente en Cuba, se renuevan los métodos y estilos de dirección empleados con el propósito de fomentar políticas encaminadas a lograr una estructura de composición de los cuadros y trabajadores, conforme a las condiciones existentes del entorno económico y político en que se desarrollan las entidades (Morales, 2009).

Para garantizar el adecuado desempeño de los trabajadores y asegurar el logro de una elevada eficiencia económica, resulta indispensable el conocimiento de las limitaciones fundamentales de estos $y$ en tal sentido, determinar las vías más adecuadas para brindarle solución y obtener la idoneidad que se requiere.

La identificación de las causas que influyen en el inadecuado funcionamiento de las organizaciones laborales tiene cada día mayor importancia, de ello depende la calidad con la que se desarrolla el trabajo, así como en la permanencia de los trabajadores en sus puestos laborales.

La fluctuación laboral — como fenómeno organizacional más frecuente en muchas organizaciones - consiste en los movimientos de entrada y salida definitivos de los trabajadores en la organización, durante un período de tiempo (Rodríguez, 2012).

Las consecuencias de la fluctuación laboral generalmente se agudizan cuando resulta extremadamente difícil ocupar el puesto de trabajo vacante, sobre todo si estos son poco atractivos y con baja remuneración, pertenecientes a empresas que proyectan una imagen negativa en el entorno donde desenvuelven su actividad. Ante esta situación, las empresas recurren a diferentes estrategias, una de estas es la de ocupar el puesto abandonado, con trabajadores que requerían previamente un período de aprendizaje y de adaptación; otros deciden sobrecargar al resto de los trabajadores con las funciones del puesto abandonado. En cualquier caso, las consecuencias se traducen en afectaciones a los niveles de eficiencia y productividad previstos para la organización. 
Un estudio realizado por estudiantes $y$ profesores de la Filial Universitaria Municipal Jesús Menéndez en la Unidad Básica de Producción Cooperativa (UBPC), evidenció la existencia de altos niveles de fluctuación laboral en la organización, aspectos que afectan el cumplimiento de su misión y los resultados económicos de la entidad.

Por lo expuesto anteriormente se identificó como problema científico: ¿cómo contribuir a la disminución de los índices de fluctuación laboral en la UBPC Luis Peña Martínez?

Se plantea como objetivo general: implementar un conjunto de acciones que contribuyan a la disminución de los índices de fluctuación laboral en la UBPC Luis Peña Martínez del municipio Jesús Menéndez.

Se determinan como tareas científicas:

1) Determinar las causas que inciden en la fluctuación de los recursos humanos en la UBPC Luis Peña Martínez.

2) Diseñar un plan de acción dirigido a la modificación de las causas que inciden en la fluctuación de los recursos humanos en la UBPC Luis Peña Martínez.

3) Evaluar los resultados preliminares obtenidos a partir de la implementación del plan de acción diseñado en la UBPC Luis Peña Martínez.

\section{MATERIALES Y MÉTODOS}

\section{DESCRIPCIÓN DE LA INVESTIGACIÓN}

Para el desarrollo de la investigación, se utilizó la información disponible en la UBPC Luis Peña Martínez en los años 2010 y 2011. La selección de la muestra se realizó a través del método aleatorio simple (Miranda, 2011), para lo que se aplicó la fórmula:

$$
n=\frac{Z_{\alpha / 2}^{2} P Q N}{\varepsilon^{2}(N-1)+Z^{2} P Q}
$$

En la que, $\mathrm{n}=$ tamaño de la muestra necesaria

$\mathrm{P}=$ probabilidad de que el evento ocurra $(0,5$ o $50 \%)$

$\mathrm{Q}=$ probabilidad de que un evento no ocurra $(0,5$ o $50 \%)$ con un error de

$\varepsilon=\operatorname{error}(0,05$ o $5 \%)$

$\mathrm{N}=$ tamaño de la población

A partir de la muestra de 139 trabajadores de la UBPC, se aplicó la técnica de la entrevista semiestructurada al segmento comprendido por los directivos y técnicos, de ellos: doce dirigentes $y$ administrativos que representó el $66,6 \%$ del total de estas categorías y ocho técnicos para un $80 \%$ del total. Por su parte la técnica de la encuesta se utilizó con 119 obreros, lo cual representó el 86\% del total.

Para el cálculo de los índices de fluctuación laboral se utilizó la fórmula (Navarro, 2004):

$\mathrm{IFLR}=\mathrm{D} * 100 / \mathrm{EM}$

Donde:

IFLR = índice de fluctuación laboral real

$\mathrm{D}=$ cantidad de egresos

$\mathrm{EM}=$ efectivo medio en un periodo determinado

\section{MÉTODOS Y TÉCNICAS EMPLEADOS EN LA INVESTIGACIÓN}

\section{MÉTODOS TEÓRICOS}

४ Observación: se empleó con el objetivo de recopilar información acerca de los elementos dinámicos presentes en las relaciones vinculares entre los diferentes factores que integran la UBPC, para ello se hizo necesario elaborar una guía de observación.

$\diamond \quad$ Análisis de documentos: empleado a lo largo de todo el proceso investigativo, con el objetivo de obtener información complementaria que permitió profundizar en el estudio del tema de investigación, contribuyendo a enriquecer los análisis y valoraciones realizadas como parte del estudio.

$\diamond \quad$ Histórico-lógico: permitió el ordenamiento cronológico de los fundamentos 
teóricos relacionados con el tema de la creación de las UBPC en Cuba, así como las tendencias actuales a nivel nacional $\mathrm{e}$ internacional en el análisis de los índices de fluctuación laboral, como herramienta para la toma de decisiones en las organizaciones laborales.

$\diamond \quad$ Análisis y síntesis: se empleó durante el análisis de la bibliografía y la posterior sistematización de toda la información consultada. De la misma forma se empleó en el procesamiento de los datos recopilados e interpretación y discusión de los resultados obtenidos durante la investigación.

$\diamond \quad$ Inducción-deducción: a partir del estudio de la bibliografía disponible, el método permitió propiciar el análisis reflexivo de la información recopilada, arribar a juicios $y$ valoraciones que facilitaron la interpretación de los resultados alcanzados en el estudio.

\section{TÉCNICAS EMPLEADAS}

$\diamond \quad$ Entrevista semiestructurada a informantes clave: esta técnica se utilizó con el propósito de conocer la percepción que poseen directivos y técnicos de la UBPC en relación con el clima organizacional, así como de las causas que influyen en la fluctuación laboral de la organización.

$\diamond \quad$ Encuesta a trabajadores de la UBPC: se utilizó con el objetivo de recopilar información entre los trabajadores en relación con el clima organizacional y las causas que conllevan a la fluctuación laboral.

Como perspectiva de análisis, se asumió la complementariedad o síntesis multimetodológica que permitió la combinación de las perspectivas cuantitativa y cualitativa. Este enfoque permitió que la investigación se realizara de forma profunda, con lo que se logró un análisis preciso de los aspectos de la realidad que son estudiados al posibilitar fusionar procesos de conocimiento mutuo. Desde esta perspectiva se admite que la investigación fue realizada con un mayor rigor científico, veracidad, confiabilidad y seguridad de los resultados obtenidos (Méndez, 2011).

Para la identificación, análisis y evaluación de los resultados preliminares (inmediatos) obtenidos durante la investigación, se aplicó el enfoque de Gestión por Resultados (GPR) (ACDI, 2009).

Desde este enfoque, se define la existencia de una cadena de resultados en los que a partir de insumos que se requieren para el desarrollo de actividades planificadas se obtiene un producto del que se derivan a su vez resultados inmediatos (a corto plazo), resultados intermedios (mediano plazo) y resultados finales (a largo plazo), elementos que fueron considerados durante la investigación.

\section{RESULTADOS Y DISCUSIÓN}

CARACTERIZACIÓN DE LA UNIDAD BÁSICA DE PRODUCCIÓN COOPERATIVA LUIS PEÑA MARTÍNEZ

La Unidad Básica de Producción Cooperativa (UBPC) se fundó el 20 de noviembre de 1993, en el Consejo Popular El Canal, ubicado al norte del municipio Jesús Menéndez, provincia Las Tunas, Cuba.

La unidad dispone de áreas de suelos y la infraestructura técnico-productiva necesaria para el cumplimiento de su misión. Además, cuenta con una fuerza de trabajo integrada por 181 trabajadores, de los cuales 130 se encuentran vinculados directamente a la producción agropecuaria. La UBPC está estructurada por la Asamblea General, a la que se subordina el Consejo de Administración, compuesto por el administrador $y$ demás integrantes. Entre los órganos colegiados de dirección - como forma cooperativa de las actividades fundamentales de la organización- se encuentran: Consejo de Dirección, Junta Económica, Asamblea de Trabajadores y Reuniones de Coordinación o Consejillos Operativos.

\section{CARACTERÍSTICAS DE LA FLUCTUACIÓN LABORAL EN} LA UBPC LUIS PEÑA MARTÍNEZ

El análisis de la fluctuación laboral real, por categoría ocupacional y periodos evaluados, mostró una tendencia al incremento en el año 
2011 con respecto al 2010, con 69 egresos más. Un análisis por etapa permitió determinar que en el 2010, la categoría ocupacional obreros obtuvo los mayores porcentajes de fluctuación con el 69,3\%, seguida de los técnicos con el $13,8 \%$ y en la tercera posición, los trabajadores de servicios con el 11, 1\% de fluctuación laboral. La categoría ocupación de más bajo índice en el 2010, fue de dirigentes con solo 1,4\% de fluctuación laboral.

Un comportamiento similar al registrado en el año 2010, se obtuvo en el 2011. La categoría de obrero registró la mayor fluctuación, seguida de los técnicos y los trabajadores de servicio, de igual forma el más bajo índice se obtuvo para los dirigentes, mientras que se registró un incremento de la fluctuación de las categorías de administrativos y técnicos, en comparación con el 2010.

En relación con el sexo, los mayores índices de fluctuación lo obtuvieron los hombres con el $64,9 \%$, lo que puede estar relacionado con la menor presencia de mujeres en la organización, a partir de que el objeto fundamental de la organización lo constituye la producción y comercialización de la caña de azúcar.

De acuerdo a los grupos de edades, el rango de edades que mayor fluctuación registró, correspondió al de 30 a 39 años, con el $46,5 \%$, seguida por el rango entre 20 a 29 años con el 29,2\% y el de menores de 20 años, obtuvo el 19,3\%. La menor fluctuación registrada, se obtuvo para el rango de 60 años $y$ más, con solo el 1,0\%. Este aspecto corresponde con la estructura etaria de los trabajadores en la UBPC.

Por su parte, el análisis de la fluctuación laboral según el nivel de escolaridad, evidenció que los mayores índices se obtuvieron para el nivel medio escolar y el de secundaria básica con el $32,8 \%$ y el $47,1 \%$ respectivamente. Este aspecto refleja el elevado nivel cultural alcanzado por la sociedad cubana a partir de la importante inversión realizada en materia de educación, después de 1959 (Nuñez, Montalvo y Pérez, 2006).
PRINCIPALES CAUSAS QUE INFLUYERON EN LA FLUCTUACIÓN LABORAL DE LA UBPC LUIS PEÑA MARTÍNEZ

$\diamond \quad$ El reclutamiento del personal sin la suficiente preparación para el desarrollo de sus funciones en la UBPC.

$\diamond \quad$ Dificultades con el clima organizacional de la UBPC que se expresa en indicadores, tales como:

- Deficiencias en los procesos de comunicación entre directivos y trabajadores.

- Inadecuados estilos de dirección con predominio del autoritarismo.

$\diamond \quad$ Insatisfacción de los trabajadores por la atención a sus necesidades por parte de sus dirigentes $y$ administrativos de la UBPC.

$\diamond \quad$ Deficiencias en la actualización y capacitación de los trabajadores de acuerdo a las nuevas demandas en la UBPC.

$\diamond \quad$ Bajo reconocimiento moral y material a los trabajadores por su desempeño laboral.

$\diamond \quad$ Condiciones laborales desfavorables $y$ falta de recursos para el desarrollo del trabajo en la UBPC.

$\diamond \quad$ Deficiencias con los sistemas de pago en la UBPC.

Este resultado corresponde con lo informado por autores como Álvarez (2001); Costa (2009) y Morales (2009), quienes informaron que la necesidad de reconocimiento moral y material, los inadecuados métodos y estilos de dirección, así como, la insatisfacción con la evaluación, constituyen causas que influyen en los índices de fluctuación laboral.

\section{EVALUACIÓN PRELIMINAR DEL PLAN DE ACCIÓN IMPLEMENTADO}

Se elaboraron un total de 15 acciones dirigidas a solucionar las causas que influyeron en los índices de fluctuación laboral registrados en la UBPC Luis Peña Martínez durante los años 2010 y 2011, a partir del mejoramiento del clima organizacional y el incremento en la efectividad y la productividad del trabajo en la organización. 
Como resultados preliminares se documentan los siguientes:

$\diamond \quad$ Se constituyó la Comisión de Admisión, como estructura de asesoramiento a la administración en materia de política de reclutamiento de los trabajadores en la organización.

$\diamond \quad$ Diseño de un sistema de trabajo para el funcionamiento de la Comisión de Admisión, de esta forma se garantiza la viabilidad y sostenibilidad de la estructura creada.

$\diamond \quad$ Realización de seis sesiones de capacitación en la UBPC, con la asistencia de 77 participantes y el acompañamiento de la Filial Universitaria Municipal Jesús Menéndez. Los temas tratados fueron: aspectos para la selección y el reclutamiento de los trabajadores, la comunicación organizacional como herramienta en las organizaciones laborales, métodos $y$ estilos de dirección para una administración eficiente, la conciliación y la resolución de problemas laborales, la evaluación del desempeño y su importancia en el cumplimiento de los objetivos y las metas de las organizaciones.

$\diamond \quad$ Se realizaran actividades recreativas en la organización dirigidas a propiciar el intercambio entre los trabajadores de la UBPC.

$\diamond \quad$ Reconocimiento de forma material $y$ moral, de aquellos trabajadores que mejores resultados productivos y laborales obtuvieron en la etapa.

$\diamond \quad$ Constitución de una brigada de mantenimiento con trabajadores de la UBPC, los que en jornadas de trabajo voluntario desarrollan labores de limpieza, embellecimiento y mantenimiento de las áreas y locales, lo que ha permitido mejorar el ambiente laboral en la organización.

$\diamond \quad$ El promedio de egresos de trabajadores por trimestre durante los años 2010 y 2011 fue de 93 efectivos; el egreso promedio registrado en la UBPC durante el primer trimestre de 2012, fue de solo 81 trabajadores, lo que sugiere un ligero decrecimiento en la tasa de fluctuación laboral de la organización en comparación con etapas anteriores.

\section{CONCLUSIONES}

La caracterización realizada en la organización permitió identificar la existencia de al menos siete causas que influyeron durante el periodo evaluado en los elevados índice de fluctuación laboral, las más importantes: deficiencias en el clima organizacional, bajo reconocimiento moral y material a los trabajadores, así como, las desfavorables condiciones laborales a las que están expuestos.

El plan de acción diseñado promovió la participación de todos los factores de la organización en la solución de los problemas que afectan a la institución, existiendo evidencias que sugieren la disminución gradual de los índices de fluctuación laboral, a partir de la transformación de las causas que influyeron en su elevada expresión.

La evaluación preliminar de los resultados obtenidos con la implementación de las acciones desarrolladas, mostró que existen potencialidades y recursos no explotados en la organización para modificar las causas que influyen en los elevados índices de fluctuación laboral y de esta forma, contribuir al incremento de los parámetros de productividad $y$ de eficiencia.

\section{BIBLIOGRAFIA}

LIBROS

Miranda, Ileana. Estadística aplicada a la sanidad vegetal. Cuba: Centro Nacional de Sanidad Agropecuaria (CENSA), 2011.

Moros, Elena y Díaz, Maiky. Selección de lecturas de Psicología Organizacional II. La Habana, Cuba: Editorial Félix Varela, 2005.

Nuñez, Jorge; Montalvo, Luis F. y Pérez, Isarelis. "Universidad, conocimiento y desarrollo local (basado en el conocimiento)". Desarrollo local en Cuba. Retos y perspectivas. Ada Guzón (comp.). Cuba. Editorial Academia, 2006: 205-219. 
TESIS

Álvarez, L. "Procedimiento de diseño de sistemas de estimulación". [Tesis de Maestría en Dirección]. Cuba. Universidad de Holguín, 2001: 18-42.

Méndez, Aliek. "Conjunto de acciones psicosociales para el fortalecimiento de la labor de prevención social en el municipio Jesús Menéndez". [Trabajo de Diploma en opción al título de Licenciado en Psicología]. Las Tunas, Cuba. Facultad de Ciencias Sociales y Humanísticas, Universidad Vladimir Ilich Lenin, 2011: 35.

Morales, Alfredo. "La integración de la gestión de los recursos humanos con la estrategia empresarial". [Tesis de Maestría]. Cuba. Ministerio del Trabajo y la Seguridad Social, 2009: 26.

Navarro, Raymundo. "Factores sociolaborales que caracterizan la fluctuación laboral". [Tesis de Maestría en Salud Pública]. Cuba. Escuela Nacional de Salud Pública, 2004: 38 .

Rodríguez, A. "Acciones para la disminución de los índices de fluctuación laboral en la Unidad Básica de Producción Cooperativa Luis Peña”. [Trabajo de Diploma en opción al título de Ingeniero en Procesos Agroindustriales]. Las Tunas, Cuba. Facultad de Ciencias Técnicas. Universidad Vladimir Ilich Lenin, 2012: 1-13.
TEXTOS ELECTRÓNICOS

Costa, Haylln. La fluctuación laboral en los procesos claves de la empresa eléctrica de Matanzas. 2009. En: <http:// www.monografias.com/trabajos $86 /$ fluctuacion-laboral-procesos-claves/ fluctuacion-laboral-procesos-claves. shtml> [consultado el 24 mayo de 2012].

Morales, Eudaldo. GRH, evolución, conceptos $y$ diferentes perspectivas vistas en la realidad cubana. 2000. En: <http:// www.gestiopolis.com/canales/derrhh/ articulos/30/grh.htm> [consultado el 18 mayo de 2011].

OTROS DOCUMENTOS

Agencia Canadiense de Desarrollo Internacional (ACDI). "Taller de capacitación en gestión de resultados TPR". Manual de los participantes. La Habana, Cuba: Le Groupe-Conseil Baastel ltée, 2009.

Espinosa, Malva; Yanes, Hugo; Espino, Alma; Abramo, Laís y Yáñez, Sonia. Inserción laboral femenina: sindicalismo, género y flexibilización en el Mercosur y Chile. Jaime Ensignia y Sonia Yañez (eds.). Santiago de Chile, Chile: Fundación Friedrich Ebert (Representación Chile) y Centro de Estudios de la Mujer (CEM), 1999.
Fecha de ingreso: 19/08/2013 Fecha de aprobación: 18/12/2013 
